\title{
Sistemas de producción tipo kanban: Descripción, componentes, diseño del sistema, y bibliografía relacionada.
}

\author{
OSCAR JAVIER PARRA ORTEGA \\ POLITÉCNICO GRANCOLOMBIANO \\ oparraor@poligran.edu.co
}

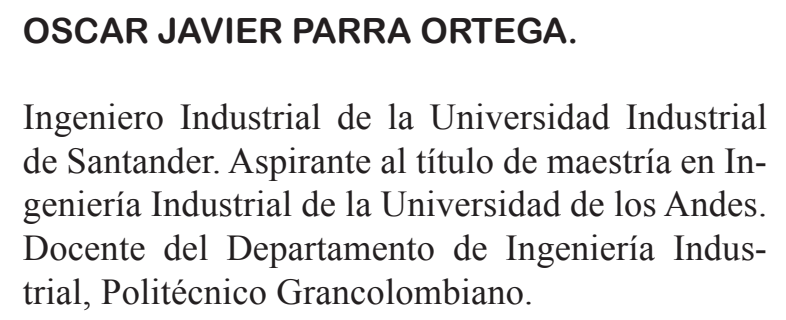

Ingeniero Industrial de la Universidad Industrial de Santander. Aspirante al título de maestría en Ingeniería Industrial de la Universidad de los Andes. Docente del Departamento de Ingeniería Industrial, Politécnico Grancolombiano.

\section{RESUMEN:}

El presente artículo tiene como objeto de estudio, los sistemas de producción tipo ensamble (tree structure) tipo Kanban, mono-producto, con tiempos de procesamiento aleatorios para las estaciones que lo conforman, y con capacidad limitada de producción para cada estación. Adicionalmente, se restringe el análisis de dichos sistemas, para horizontes finitos de producción. Después de una descripción del mecanismo de operación de un sistema Kanban simple, de los elementos que los componen, y del estado del arte en sistemas de producción tipo Kanban, se propone e implementa un modelo heurístico para determinar el número de kanbans, minimizando el costo promedio del inventario en proceso.

\section{ABSTRACT:}

The present article has as study object, the Kanban type assembly production systems (tree structure), mono-product, with random times of processing for the stations that conform it, and with limited capacity of production for each station. Additionally, the analysis of these syste$\mathrm{ms}$ is restricted, for finite horizons of production. After describing the mechanism of operation of a single card Kanban system, as well as their components, and review studies related to Kanban production systems, it proposes and implements a heuristic model to determine the number of kanbans, minimizing the average cost of the inventory in the process.

\section{PALABRAS CLAVE:}

Sistemas Kanban; heurísticas; simulación; líneas de ensamblaje

\section{KEYWORDS:}

Kanban Systems; heuristics; simulation; assembly lines 


\section{INTRODUCCIÓN}

Kanban es un componente esencial de la filosofía de gestión de operaciones JIT (just in time), la cual tiene como orientación básica, la reducción del nivel de inventarios, mediante procesos que satisfagan la demanda en la cantidad, y en el tiempo requeridos. Para implementar esta filosofía, propone la aplicación de un sistema de control de producción tipo Pull, en el cual los ítems son producidos al ritmo que se necesitan, y donde la última estación es la que marca dicho ritmo, haciendo reposición de las unidades consumidas. De esta forma, una estación produce únicamente como respuesta a una orden de reposición inmediatamente posterior de ítems consumidos por su estación. El sistema tipo Pull más conocido es el tipo kanban, en el cuál las ordenes de reposición de ítems consumidos se transmiten a través de señales conocidas como kanbans.

En su traducción literal, la palabra japonesa kanban significa "señal" o "tarjeta". En el contexto de producción, se refiere específicamente a un sistema de señales visuales de control de producción que autoriza o dispara (trigger) el proceso de reabastecimiento. Esta señal de reabastecimiento puede tomar una amplia variedad de formas, desde tarjetas o tableros, hasta señales electrónicas (e-kanban). La decisión respecto a la forma que esta señal va a tomar dependerá de diversos factores, tales como la distancia que tiene que recorrer la señal, y el contenido que debe transmitir. A continuación se describirá el funcionamiento de este mecanismo, en su acepción clásica, los componentes del sistema en su fase de diseño, y finalmente se hará mención de la bibliografía disponible hasta la fecha sobre el tema para un estudio más profundo.

\subsection{Descripción del sistema tipo kanban}

Inicialmente, se hará precisión sobre los elementos que delimitan, o que caracterizan los sistemas de producción tipo kanban.

Una instalación de manufactura está conformada por una serie de estaciones de trabajo conectadas entre sí por relaciones de precedencia en la realización de operaciones, y por el flujo de ítems e información asociada con la realización de dichas operaciones. Cada una de estas estaciones de trabajo tiene la siguiente configuración: un número de servidores (los cuales pueden ser máquinas, operarios, o una combinación de estos) que trabajan en paralelo dentro de la estación, y que realizan una secuencia de actividades sobre los elementos que se van a procesar. Cada estación está caracterizada por:

-La distribución de su tiempo de procesamiento.

-El tamaño de lote que procesa.

-Un conjunto de estaciones predecesoras que le suministran ítems para procesar.

- Un mecanismo de activación del proceso de producción dentro de la estación, y otro mecanismo de activación para el transporte de ítems terminados a las estaciones posteriores.

Cada una de las estaciones así descritas, maneja en un instante determinado, dos tipos de ítems: terminados, y en proceso, clasificados a su vez como componentes, materias primas, ensambles o subensambles. Una vez han sido procesados, los ítems terminados se agrupan en lotes de un tamaño determinado (tamaño de lote de transferencia) y son transportados a las estaciones posteriores en la instalación de manufactura para continuar con la secuencia de procesamiento. El tamaño del lote de transferencia dependerá tanto de las características de la estación que envía los ítems, como de la que los recibe.

A su vez, las estaciones se pueden describir bajo las siguientes relaciones de precedencia:

-Toda estación que recibe ítems terminados tipo i, se considera posterior inmediata a $i$.

-Toda estación que envíe ítems terminados a la estación i, se considera predecesora inmediata de i.

Con base en las definiciones anteriores, a continuación se muestra el tipo de configuración conocido como kanban simple.

\subsubsection{Kanban simple (SK)}

Esta configuración utiliza un tipo de tarjetas conocidas como kanban de transferencia (kanban de retiro). Cada kanban de transferencia se utiliza para comunicar la necesidad de transferir un contenedor lleno de materia prima, desde una estación predecesora (upstream) i, hacia la siguiente estación (downstream) i +1 . Todas las estaciones están provistas de un buffer de kanbans entrantes y otro buffer de kanbans salientes, así como un punto de almacenamiento de material entrante, y un punto de almacenamiento de material saliente.

$\mathrm{Al}$ inicio de cada periodo de reabastecimiento, las tarjetas 
almacenadas en el buffer de kanbans salientes de la estación $i+1$, se llevan al buffer entrante de la estación i. Estos kanbans van a actuar en la estación i como señales de activación del proceso de reabastecimiento, y se ubican en el mismo orden en el que venían de la estación $i+1$.

A su vez, al inicio de cada ciclo de reabastecimiento y retiro (withdrawal cycle), los contenedorcontenedores con material procesado en la estación i, son transferidos al punto de almacenamiento de material entrante de la estación $i+1$ (se efectúa el proceso de reabastecer a la estación $i+1)$. Nótese que cada uno de estos contenedorcontenedores tiene adjunto un kanban.

El proceso de producción en la estación i da inicio únicamente cuando tenga un kanban en el buffer entrante i, y cuando haya material disponible en el punto de almacenamiento de material entrante de dicha estación. Cuando se cumplan estas dos condiciones, el proceso de producción iniciará, y el kanban que tiene adjunto el contenedor de materia prima que se empieza a utilizar es retirado y ubicado en el buffer saliente.

Finalmente, cuando un contenedor en i se ha completado, se le adjunta el kanban que generó su producción (el que estaba ubicado en el buffer entrante i). El container completo se ubica en el punto de almacenamiento de material saliente, para ser transferido a la estación $\mathrm{i}+1$, al inicio del siguiente ciclo de reabastecimiento.

\section{Componentes del diseño de un sistema kanban}

El sistema de reabastecimiento kanban puede considerarse como uno de los más sencillos de entender en su filosofía, pero uno de los más difíciles de aplicar de forma exitosa. Su efectividad depende principalmente de dos factores: que sea diseñado para cumplir con las necesidades específicas del entorno bajo el cual va a operar, y que sea implementado de forma apropiada. Es un error común intentar replicar de forma exacta las técnicas propuestas por un autor determinado o las prácticas exitosas vistas en otras plantas de producción, sin seleccionar debidamente la técnica o técnicas kanban que mejor se adapten a los factores específicos del entorno de producción. Entre otros factores para tener en cuenta, se pueden enumerar los siguientes:

-Expectativas del cliente final frente a la capacidad de respuesta.
- Comportamiento de la demanda.

- Cantidad de partes activas en el sistema.

-Ubicación y capacidad de respuesta de los proveedores

Un sistema kanban diseñado a la medida del entorno de producción genera las cantidades de producción en los momentos en que se requieren, y está acompañado por una visible reducción de inventario, faltantes, actividades que no generan valor agregado, y de los costos globales de fabricación. Si funciona debidamente, este sistema requerirá un menor nivel de intervención y esfuerzo para su funcionamiento, e incrementará el porcentaje de órdenes entregadas a tiempo.

En cambio, si el diseño no es el apropiado, la brecha subyacente entre el sistema de abastecimiento y los requerimientos del entorno de producción va a manifestarse en un mayor nivel de inventario, un sobredimensionamiento del tamaño de lote kanban, y en la aparición de listas con prioridad de entrega (para responder a las órdenes retrasadas que suelen aparecer).

Mecanismo de gestión de la demanda final. Este componente es vital para reducir el impacto de los patrones de demanda no lineales que puedan afectar la eficiencia del sistema kanban. Incluyen, pero no se limitan, a técnicas de pronóstico y de ajuste de la demanda con la capacidad existente del sistema.

Tipo de contenedor que se va utilizar. Vienen determinados de acuerdo con el tamaño y peso de los componentes, el volumen de la demanda, y la distancia entre estaciones.

Dimensionamiento de lote kanban. Debe establecerse teniendo en cuenta el comportamiento de la demanda, la disponibilidad y condiciones de suministro de los proveedores, así como los tiempos de preparación para cada estación.

Mecanismos de activación. Son aquellas reglas de decisión que dan pie al proceso de producción y reabastecimiento de inventario entre estaciones. Su elección y configuración depende de la cantidad de partes, del tamaño de la planta, y de la integración con sistemas MRP (cuando coexisten con el sistema $\mathrm{k}$.

Mecanismos de intervención. Como respuesta a las variaciones en la demanda, estos mecanismos se encargan 
de ajustar el número de tarjetas (kanbans) que circulan en el sistema.

Manejo de materiales. De acuerdo con estos mecanismos, se pueden reducir sustancialmente los costos indirectos asociados con la inspección y movimiento de materiales entre estaciones.

Interacción con proveedores. Con el fin de integrar la cadena de abastecimiento al sistema de producción, deben tenerse en cuenta como restricciones del sistema, los tiempos de reabastecimiento (lead time) de los proveedores, y el tipo de ítems requeridos como suministro para iniciar el proceso de producción.

\subsection{Mecanismos de gestión de la demanda fi- nal.}

Un requisito esencial para alcanzar la eficiencia en un sistema kanban de manufactura, es lograr que el comportamiento de la demanda de productos terminados tienda a ser lineal. Si por el contrario, el comportamiento de la demanda es errático, el sistema de producción tiende a verse afectado de forma negativa a causa de las variaciones súbitas en el nivel de producción. Problemas de calidad, mayor dificultad para mantener la confiabilidad de la maquinaría de producción, incremento de inventario, y de los costos de producción, pueden ser provocados por la ausencia de mecanismos apropiados para gestionar el comportamiento de la demanda.

Por lo anterior, en la primera fase del diseño de un sistema de producción kanban es primordial seleccionar, tanto los mecanismos de programación de la producción final, como los mecanismos de actualización de los pronósticos de la demanda.

Entre los mecanismos de programación de la producción sugeridos por Raymond [39] para los sistemas kanban, se tienen los siguientes:

-Un plan maestro de producción (Master Production Schedule), que indique las cantidades del producto final que se va a fabricar o ensamblar en cada periodo de tiempo, con base en las órdenes actuales y en las pronosticadas. Se recomienda aplicar un MPS de suavizamiento de carga (load-smoothing) el cuál desarrolla una programación diaria de producción a partir de una relación lineal entre el pronóstico para el periodo de producción y el número de días hábiles del periodo. Para el caso de líneas de fabricación multiproducto, además se debe determinar la secuenciación de productos de forma que se asegure, en el curso del día, un patrón de demanda cíclica lineal entre las distintas referencias a producir.

Esta alternativa se sugiere en los casos en los cuales la demanda no tiene un comportamiento lineal, y cuando la capacidad de respuesta del sistema supera los requerimientos de demanda de los clientes finales.

-Activación de la producción en la última estación de la línea de ensamble, a partir de la llegada de nuevas órdenes -Para líneas de ensamble multiproducto donde se combinan productos de demanda estable (y alto volumen) junto a otros con demanda errática y bajo volumen de ventas, se utiliza de forma conjunta el sistema kanban para los productos de alto volumen, y la activación por órdenes de producción para los ítems de demanda errática y bajo volumen. La prioridad para el ingreso a la celda de producción de los ítems de baja demanda, se asigna de acuerdo con las expectativas de entrega acordadas por el cliente.

-Para mezclas de productos con componente estacional en el comportamiento de su demanda, se recomienda establecer un MPS para la planeación de la producción, junto con la activación por órdenes de producción exclusivamente para productos de bajo volumen.

-Para productos altamente personalizados, la opción más apropiada es la de iniciar la producción con base en las especificaciones de cada orden, pero generar un MPS para la planeación de capacidades en los modelos básicos (genéricos) de la línea.

- Finalmente, para sistemas de producción tipo taller (job shop) con comportamiento no lineal en la demanda, se recomienda aplicar kanban exclusivamente en los niveles inferiores de producción (materias primas y algunos subensambles y ensambles) en los cuáles exista alta similitud.

Ahora, el segundo aspecto de importancia en el diseño de los mecanismos de gestión de la demanda final, es el relacionado con los pronósticos y con la forma como estos 
alimentan al MPS para la planeación de la capacidad. Se recomienda utilizar uno de los siguientes mecanismos - Generar pronósticos que reflejen directamente las cantidades de cada ítem por periodo de planeación. Es eficiente si se maneja un número reducido de productos finales.

- Generar pronósticos en unidades monetarias, y automatizar el proceso de conversión de dichos pronósticos en términos de cada referencia y periodo de tiempo. Es recomendable para sistemas que manejan un alto número de referencias de producto terminado.

\subsection{Tipo de contenedor que se va a utilizar}

De acuerdo con el tipo de ambiente de producción, y con las características asociadas a cada producto (volumen de demanda y costos asociados), se debe seleccionar apropiadamente el tipo de contenedor Kankan que se va a utilizar. Existen cinco tipos de contenedor diferenciadoscontenedor:

- Container sencillo de cantidad unitaria (discreto). Se utiliza para mover ítems que por sus grandes dimensiones y alto costo no permiten el manejo de contenedorcontenedores de capacidad múltiple. En lugar de utilizar un contenedor físico, el sistema kanban maneja un espacio demarcado para la utilización (procesamiento) del ítem.

La activación de reaprovisionamiento (triggering) se genera con base en el inventario de piezas disponibles más las solicitadas (on-order + on-hand). De todas las alternativas, esta es la que tiende a manejar el menor nivel de inventario promedio, a causa del alto costo de los ítems. El inconveniente que presenta este tipo de contenedor es que por manejar un menor inventario promedio, la estación a la cual alimenta (estación posterior) es más sensible al tamaño de lote seleccionado y pueden presentarse faltantes. Por eso es recomendable aplicar este tipo de contenedor a subensambles o ensambles terminados, o productos finales, más que a partes que alimentan a otras estaciones. Nótese que el tamaño del contenedor para este caso es 1 , y que el número de contenedorcontenedores equivale al tamaño de lote kanban.

Container sencillo de capacidad total. Se utiliza para mover y reaprovisionar ítems de bajo costo, en los cuales el costo de transporte del contenedor es relevante frente al costo de los componentes que alberga, y por esta razón, no hay desplazamiento físico de contenedorcontenedores entre estaciones. Los componentes son de pequeño tamaño y el tiempo de preparación es relevante para calcular las cantidades de reaprovisionamiento (tamaño de pedido medido como en términos del número de contenedorcontenedores por solicitar para reaprovisionar a la estación a la que sirve).

El mecanismo de activación en este caso es el siguiente: si el número de piezas disponibles más piezas solicitadas (inventory on-hand + inventory on-order) es inferior al tamaño de lote kanban, se solicita un número de piezas equivalente al tamaño de lote kanban. Por ejemplo, si el tamaño de lote kanban es de 80 unidades y hay disponibles 79, se hace una orden de reaprovisionamiento por 80 piezas. Es claro que este mecanismo requiere que el conteo de inventario disponible, así como el lanzamiento de órdenes de reaprovisionamiento sea automatizado.

-Contenedores duales. Este se utiliza también para productos pequeños, pero con la salvedad de que el tamaño de lote kanban puede fluctuar entre periodos de planeación. Funciona de la siguiente forma: hay dos contenedor idénticos uno detrás de otro en una estación de procesamiento. Solo cuando el container frontal está vacío, el container posterior avanza y el vacío se envía a la estación precedente (o al proveedor para su reaprovisionamiento). Una vez se ha hecho el reaprovisionamiento del container, este se retorna a su estación de uso.

Tal como sucede con los contenedor sencillos de capacidad total, y con los contenedor triples, se maneja un tamaño de lote kanban para la activación del reaprovisionamiento, dado que el tiempo de preparación en la estación precedente es relevante.

- Contenedores triples. Se utiliza únicamente para reaprovisionamiento externo, cuando el proveedor está ubicado a una distancia considerable del sistema de producción. La activación se genera por tamaño de lote kanban, y el sistema funciona de la siguiente forma: hay dos contenedores idénticos en el punto de uso (estación), y un tercer contenedor vacío está en la ubicación del proveedor del material para dicha estación. En cuanto se vacía el contenedor frontal, este inicia la activación de la orden hasta que se alcance el tamaño de lote kanban. La señal de activación se hace de forma electrónica o (EDI, Extranet o 
FTP, entre otros) de forma que el kanban de producción (el tercer P-kanban vacío que está en la ubicación del proveedor) se activa sin que haya demora de transporte del primer contenedor vacío desde la estación hacia el fabricante de material.

-Múltiple manejo de tarjetas en el sistema de contenedor. Esta configuración utiliza dos o tres tipos de tarjetas, que prestan diferentes funciones para la activación de procedimientos en sistemas con múltiples contenedores. A continuación se describen ambas variantes:

-Enfoque clásico de tres tarjetas. Se maneja una tarjeta de producción (P-kanban), una tarjeta de retiro de material (T-kanban), y una tarjeta para proveedores (S-kanban). Las tarjetas de producción generan en las estaciones precedentes el proceso de producción y reabastecimiento de un nuevo contenedor. Una vez el container está lleno, hasta que no haya una T-kanban (withdrawal kanban) no se efectúa su transporte hasta el punto de uso. Las tarjetas para activación de reabastecimiento por parte del proveedor o S-kanban indican adicionalmente el ciclo de entrega (número de entregas/frecuencia de entrega/número de periodos durante los cuales está activo el reabastecimiento).

-Enfoque de dos tarjetas. Para el manejo interno en la planta, cada parte o pieza tiene asignados dos tipos de tarjeta: P-kanban y T-kanban.

Cualquiera de los mecanismos mencionados anteriormente tiene como objetivo básico el control de forma separa$\mathrm{da}$, del número de tarjetas tipo $\mathrm{P}$ y tipo $\mathrm{T}$ que circulan por el sistema. El anterior modelo sigue la recomendación de Ardalan de que las partes requeridas por una T-kanban deben ser producidas primero que las requeridas por las tarjetas de producción (una T-kanban representa una orden más urgente que la activada por una P-kanban).

\subsection{Implementación de los mecanismos de activación (triggering)}

De acuerdo con el número de referencias y partes por manejar, su tamaño y peso, y al tipo de configuración de producción, se tienen las siguientes alternativas para la implementación de los mecanismos de activación:

-Activación manual, o activación por tarjetas. Aplica- ble para sistemas con una cantidad limitada de partes, un tamaño relativamente pequeño de planta, y proveedores locales. No se recomiendan para contenedores sencillos (discretos y de capacidad total) a causa de los cálculos y controles que estos requieren para la activación de una orden

-Activación automatizada. Elimina actividades asociadas con el proceso manual de activación, ya que estas no agregan valor al producto final. Es ideal para sistemas complejos o con gran número de referencias y se acompasa con relativa facilidad a entornos que operan bajo una mezcla de ítems producidos bajo kanban y MRP.

-Metodología de emisión. Para ítems de alto costo y/o tamaño, se recomienda que el inventario disponible que mantiene cada estación sea consumido durante el periodo de producción. La orden se emite de forma que el reabastecimiento sucede en el punto de uso en el instante preciso en el cuál se requiere su ensamble (y no antes como puede pasar con las otras metodologías, en las cuales sí hay una cantidad predeterminada de inventario disponible).

-Activación por tableros o por cartas kanban. Simplifica la aplicación cuando el tamaño o peso del artículo favorece el uso de tableros en lugar de otros instrumentos que se fijan directamente al producto.

-Activación por kanbans visuales. Es conveniente cuando hay proximidad entre las estaciones (proveedora y usuaria de los ítems). El mecanismo de transporte también puede hacer las veces de kanban visual (banda transportadora, por ejemplo).

\subsection{Dimensionamiento del lote kanban}

Finalmente, el tamaño del lote kanban está asociado de forma directamente proporcional, con la demanda esperada en el periodo de producción (diaria, por hora, o por turno de producción), así como con el tiempo de reaprovisionamiento de la estación precedente (más el inventario de seguridad, expresado en unidades de tiempo como una fracción del periodo de producción).

Por ejemplo, si la producción planeada para el periodo de producción (día) es de 160 unidades, el sistema opera durante 8 horas diarias, y el lead time de la estación que alimenta a un contenedor es de una hora, se requiere que 
el tamaño del lote, por lo menos, sea de 20 unidades, más las unidades que se estimen de acuerdo con el inventario de seguridad asociado con la estación precedente.

Ahora, dividiendo el tamaño de lote kanban entre la capacidad estándar del contenedor (standard container quantity) se calcula la cantidad de pedido kanban, la cual se expresa en términos del número de contenedores. De modo que, para estaciones que operan con un solo contenedor discreto, el tamaño de lote equivale al número de contenedores unitarios que puede albergar (el movimiento entre estaciones tiene una cantidad de pedido igual a uno). En cambio, la cantidad de pedido kanban en los mecanismos de contenedores restantes (contenedor sencillo de capacidad total, doble, triple o múltiple), está ligado al tamaño estándar de cada contenedor.

\section{III.Estudios publicados sobre el análisis y de- scripción de los sistemas kanban}

Dado que existe una amplia bibliografía relacionada con los sistemas de producción tipo Pull, entre ellos los sistemas tipo kanban (con sus diversas variantes), es importante resaltar que los artículos de investigación disponibles a la fecha, se pueden clasificar dentro de cuatro categorías bien diferenciadas. La primera de ellas corresponde a aquellos estudios en los cuáles se parte del supuesto básico que los tiempos de procesamiento y de llegadas de materia prima al sistema, son conocidos y con varianza cero. Estos modelos, de tipo determínistico, conforman la mayor parte de la bibliografía disponible en cuanto a modelos de sistemas de producción acordes con la filosofía JIT.

Un segundo grupo corresponde a aquellos estudios que han incluido el modelaje de los tiempos de llegadas y/ o servicios al sistema, a partir de distribuciones de probabilidad de diversa complejidad. En esta categoría se trabaja el problema inicialmente con distribuciones tipo exponencial o Erlang, y en algunos casos a partir de cadenas markovianas simples. En la bibliografía revisada hasta el momento ningún autor cita las distribuciones tipo fase para el modelaje del sistema, a pesar de que estas distribuciones presentan ventajas desde el punto de vista geométrico matricial para los cálculos de las medidas de desempeño, y que pueden representar virtualmente cualquier tipo de distribución asociada con el comportamiento real del tiempo de proceso de una máquina en un sistema tipo ensamble. Este grupo incluye lo que se conoce como modelos estocásticos o probabilísticos.

En una tercera categoría, aparecen con mayor frecuencia en los años más recientes, diversos artículos que proponen modelos de simulación para comparar el desempeño de los sistemas tipo ensamble bajo distintas condiciones de operación. A diferencia de los modelos anteriores, en estos estudios no se optimiza el desempeño, ya que no se propone una formulación matemática cerrada para el sistema.

Finalmente, un pequeño porcentaje de los artículos encontrados se enfocan o bien en describir las generalidades y los aspectos cualitativos de los sistemas de producción tipo kanban, o en realizar una revisión de artículos orientados a aspectos específicos, como la implantación del sistema JIT y kanban en las empresas en Occidente. A continuación se resaltan los aspectos más relevantes de los artículos disponibles en la bibliografía, en orden cronológico:

Ohno(1977) hace una descripción cualitativa del sistema kanban introducido por él mismo en Toyota, y describe las características distintivas de este sistema frente a los propuestos para sistemas tipo Push: la producción JIT, orientada a fabricar los productos indicados en las cantidades precisas y los periodos necesarios de tiempo; y el respeto por el ser humano, bajo el cual todos los operarios puedan desarrollar al máximo sus capacidades a través de la participación activa en el mejoramiento de las operaciones.

Burns (1978) investiga el proceso de respuesta dinámico de una cadena de suministro multietapa, con reglas de decisión individuales para cada etapa, frente a un modelo que ajuste problemas de "falsas órdenes" generados con las reglas de decisión individuales. Ambas propuestas son evaluadas con un modelo de simulación.

Tabe y otros (1980) modela la variabilidad en las cantidades por movilizar entre etapas en un sistema tipo kanban multietapa, basándose en pronósticos frente a las fluctuaciones de la demanda, de forma que se reduzcan las variaciones del nivel de inventario en cada etapa.

Schonberger (1983) presenta las aplicaciones de las técnicas de kanban de tarjeta sencilla y tarjeta dual, y compara dichos sistemas frente a los tradicionales MRP, punto de reorden y otras técnicas de reabastecimiento continuo. Se hace énfasis en el modelo kanban dual.

Huang (1983) resalta la importancia del ambiente bajo el 
cual se va a implantar un sistema kanban y realiza una simulación para un sistema multilínea y multietapa para determinar su capacidad de adaptación a los ambientes de producción occidentales. Se modelan los problemas de balance entre las distintas etapas de producción.

Davis (1985) desarrolla un estudio sobre las dificultades que se presentan al momento de proponer un modelo de optimización discreto, que no captura con facilidad el comportamiento estocástico de diversos elementos de un sistema tipo kanban. Se desarrolla una estrategia de control para un fabricante de puertas bajo pedido, combinando simulación y optimización discreta.

Bitran y Chang (1987) proveen una formulación entera no lineal para un sistema de producción de ensamble tipo árbol, multietapa, de un solo ítem, de tipo determínistico. Esta formulación asume un tiempo cero de transporte y períodos finitos de planeación, para calcular el mínimo número factible de kanbans. El modelo inicial se transforma en un modelo de tipo lineal, en el cual no hay ningún tipo de incertidumbre en los parámetros modelados.

Philipoom (1987) hace un análisis comparativo de las características presentes en las compañías japonesas que han implantado los sistemas de producción tipo kanban, y resalta que dichas características no están presentes en casi ninguna firma occidental. Concluye que, sin un ambiente apropiado de producción, la implantación de JIT es poco probable que alcance los objetivos propuestos. Resalta los factores que influyen en el número de kanbans requeridos por estación en un entorno de producción occidental: el Throughput; los coeficientes de variación de los tiempos de procesamiento y la utilización de las máquinas. El impacto de estos factores se analiza a través de un modelo de simulación.

Krajewski y otros (1989) delimitan diversos factores esenciales para alcanzar la efectividad con sistemas tipo kanban en empresas japonesas. Los diversos niveles asignados a cada planta bajo estudio fueron incluidos para realizar los ajustes de configuración en un modelo de simulación de los sistemas de producción. A partir de una variación en dichos ajustes de configuración, se demuestra que mientras kanban mejora el desempeño en muchas plantas, existe un grupo de plantas con un comportamiento similar al de las fábricas en los EUA, para los cuáles la simulación arroja pésimos resultados en términos de desempeño. Se concluye después de variar los niveles de inventario y de confiabilidad de las máquinas, que estos factores no tiene un impacto tan elevado como el ambiente de manufactura seleccionado, en el desempeño del sistema.

Sarker (1988) trata más a fondo el efecto del desbalance entre los tiempos de operación de las etapas que conforman un sistema de producción JIT, a través de un modelo de simulación.

Gravel y otros (1988) muestran que el método kanban puede adaptarse para su aplicación en sistemas tipo jobshop. Dicha adaptación se implementa y aprueba de forma intensiva por medio de simulación.

Villena (1988) examina un sistema de ensamble tipo árbol en el cuál diversas líneas de subensamble alimentan una estación final. En la asignación de la carga de trabajo en las estaciones, se propone reducir los efectos generados por la variabilidad en los tiempos de operación. Se propone uno de los diversos métodos de desbalance para reducir la variabilidad e incrementar el Througput del sistema, y se compara dicha productividad frente a la obtenida por sistemas perfectamente balanceados. Se analiza también el efecto del tamaño permitido para el buffer entre estaciones respecto a la mejora en la productividad.

Funk (1988) discute algunos problemas asociados con el modelaje de manufactura JIT, y describe algunos modelos sencillos de costos de inventario para evaluar estrategias de reducción de costos en un sistema de ensamblaje (PCB).

Gupta(1989) utiliza un modelo de simulación dinámico para investigar las características únicas de los sistemas kanban, bajo la presencia de factores exógenos al sistema.

En Uszoy (1990), se realiza una revisión de los modelos tipo Kanban basados en sistemas de un solo ítem, y se proponen futuras direcciones para la investigación de sistemas multi ítem, procedimientos de solución heurística, análisis por escenarios y otros ambientes menos convencionales.

Hopp \& Spearman(1990) describen un sistema de producción novedoso para su época: el sistema tipo CONWIP. Se muestran las ventajas de este sistema frente a otros sistemas tipo Push y tipo Pull de forma teórica y a través de simulación.

Onvural(1990) realiza una revisión minuciosa de los distintos tipos de redes cerradas con bloqueos, entre los cuales se encuentran los sistemas tipo Kanban. La revisión está asociada a redes cerradas con colas finitas.

Graham(1992) desarrolla un modelo Markoviano de esta- 
do estable para calcular el número de kanbans en la etapa de control de los procesos que alimentan a las lineas de ensamblaje, el cuál, combinado con una política de control del número de kanbans, reduce el inventario y mejora el desempeño frente a los sistemas Kanban tradicionales. Berkley(1993) establece un método para calcular los mínimos niveles de desempeño requeridos para garantizar una tasa deseada de producción. Con base en esos niveles de desempeño, se resuelve el problema de calcular el número de kanbans en cada estación.

Chang (1994) plantea una function objetivo multi-atributo que de forma simultánea busque minimizar el tiempo de ciclo, el costo de operación y las pérdidas de capital, al optimizar el número de kanbans y de tamaños de lote. Se propone un algoritmo de recocido simulado frente al algoritmo multi-atributo, y se aplica un heurístico para mejorar el desempeño del algoritmo propuesto. Igualmente plantea en otro estudio del mismo año un modelo de simulación en el que obtiene que el sistema Kanban genérico supera en desempeño a los sistemas CONWIP. Chao(1995) propone un método de prioridad-K para lineas de producción no seriales con diferentes prioridades para sus productos. Utilizando un proceso de decisión markoviano y conceptos de programación dinámica, se desarrolla y se resuelve un modelo para sistemas Kanban con prioridades, el cuál mejora el desempeño frente a reglas de colas tipo FCFS.

Price(1995)et.al. resalta la importancia de la selección del número de kanbans a utilizar, así como el tamaño del lote-kanban, y propone un modelo de optimización para determinar el número óptimo de kanbans en un taller de ensamble y se comprueba que existe una relación entre el makespan de una orden y el número de kanbans para el taller de ensamble.

Huang(1996) actualiza la revisión de los estudios disponibles para el cálculo del número de kanbans, bajo los enfoques determínistico, probabilístico y de simulación.

Hemamalini(2000) et.al. plantean un algoritmo heurístico para calcular los tamaños de contenedor, su cantidad, y la programación de los mismos para controlar el material entre estaciones y el nivel de operación en cada estación. El algoritmo calcula el tamaño de los contenedor para minimizar las tardanzas ponderadas de los contenedor y de las ordenes de producción. Se generaron problemas aleatorios de diversos tamaños para evaluar el desempeño del heurístico.

Suri(2003) establece un sistema híbrido push-pull para el control de producción que combina los aspectos de los sistemas kanban con los tradicionales MRP. Se muestran las mejoras obtenidas con este nuevo sistema frente al throughput, la reducción del WIP y los tiempos de aprovisionamiento, así como el incremento en el porcentaje de entregas dentro de los plazos establecidos.

Finalmente, Matta(2005) desarrolla una metodología para evaluar el desempeño de sistemas kanbans multietapa que funcionan con diferentes políticas de control. Se utilizan para ello diversas técnicas de redes de colas, aplicadas a sistemas de ensamble independiente versus sistemas de ensamble simultáneo. Matta propone una política de control apropiada en la fase de diseño del sistema, en la cual los resultados se validan a través de simulación. 


\section{BIBLIOGRAFIA}

-Akturk, M. S.Erhun, F. An overview of design and operational issues of Kanban systems. International Journal of Production Research, 1999.

-Ashburn, A. Toyota's famous ohno system. American Machinist, 1986.

-Askin, R. G.Mitwasi, M. G.Goldberg, J. B. Determining the number of kanbans in multi-item just-in-time systems. IIE Transactions, 1993.

-Bard, J. F.Golony, B. Determining the number of kanbans in a multiproduct, multistage production system. International Journal of Production Research, 1991.

-Berkley, Blair J. A simulation study of container size in two-card Kanban system. International Journal of Production Research, 1996.

-Berkley, Blair J.Kiran,A. S. A simulation study of sequencing rules in a Kanban controlled flow shop. Decision Sciences, 1991.

-Berkley, Blair J. Simulation tests of FCFS and SPT sequencing in Kanban systems. Decision Sciences, 1993.

-Berkley, Blair J. A review of the Kanban production control research literature. Production and Operations Management, 1992.

-Berkley, Blair J. Setting minimum performance levels for two-card kanban-controlled lines. International Journal of Production Research, 1993.

-Berkley, Blair J. Effect of buffer capacity and sequencing rules on single-card Kanban system performance. International Journal of Production Research, 1993.

-Berkley, Blair J. Testing minimum performance levels for kanban-controlled lines. International Journal of Production Research, 1900.

-Bitran, Gabriel R.Chang, Li. A mathematical programming approach to a deterministic Kanban system. Management Science, 1987.

-Burns, J. F.Sivazlian, B. D. Dynamic analysis of multi-echelon supply systems. Computers \& Industrial Engineering, 1978.

-Buzacott, J. Queueing Models of Kanban and MRP controlled production systems. Engineering Costs and Production Economics, 1989.

-Chang, T.M.Yih, Y. Generic Kanban systems for dynamic environments. International Journal of Production Research, 1994.

-Chang, T.M.Yih, Y. Determining the number of kanbans and lotsizes in a generic Kanban system: a simulated an- nealing approach. International Journal of Production Research, 1994.

-Chao, X.Das, S.K.Nagendra, P. Prioritization of kanbans in the case of a single station serving multiple downstream stations. International Journal of Production Research, 1995.

- Chaudhury, AbhljitWhinston, Andrew B. Towards an adaptive Kanban system. International Journal of Production Research, 1990.

-Cimorelli, Stephen. Kanban for the supply chain. Fundamental practices for manufacturing management. Productivity Press, NY., 2006.

-Davis, Wayne J.Stubitz, Steven J. Configuring a Kanban system using a discrete optimization of multiple stochastic responses. International Journal of Production Research, 1987.

-Deleersnyder, Jean-Luc.Hodgson, T. J.et.al. Kanban controlled pull systems: an analytic approach. Management Science, 1989.

-Funk, Jeffrey L. A comparison of inventory cost reduction strategies in a JIT manufacturing system. International Journal of Production Research, 1989.

-Graham, I. Tandem queues and kanban-controlled lines. International Journal of Production Research, 1991.

-Graham, I. Comparing trigger Kanban control of flowline manufacture. International Journal of Production Research, 1992

-Gravel, Marc.Price, Wilson L. Using the Kanban in a job shop environment. International Journal of Production Research, 1988.

-Gupta, Yash P.Gupta, Mahesh C. A system dynamics model for a multi-stage multi-line dual-card JIT-Kanban system. International Journal of Production Research, 1989. -Hemamalini, B. Determination of the number of contenedor, production kanbans and withdrawal kanbans; and scheduling in Kanban flowshops. Part I \& II. International Journal of Production Research, 2000.

-Huang, C. C.Kusiak, A. Overview of Kanban systems. International Journal of Computer Integrated Manufacturing, 1996.

-Huang, P.Y.Rees, Loren P.et.al. A simulation analysis of the japanese just-in-time technique (with Kanbans) for a multiline, multistage production system. Decision Sciences, 1983.

-Karmarkar, U.S. Kanban systems. The Graduate School of Management, U. of Rochester, 1986. 
-Karmarkar, U.S.Kekre, S. Batching policy in Kanban systems. Journal of Manufacturing Systems, 1987.

-Kim, Tae-Moon. Just-in-time manufacturing system: a periodic pull system. International Journal of Production Research, 1985.

-Kimura, Osamu.Terada, Hirosuke. Design and analysis of Pull System, a method of multi-stage production control. International Journal of Production Research, 1981.

-Krajewski, Lee J.King, Barry E.et.al. Kanban, MRP, and shaping the manufacturing environment. Management Science, 1987.

-Lee, L.C. Parametric appraisal of the JIT system. International Journal of Production Research, 1987.

-Lee, L.C. JIT and the effects of varying process and setup times. International Journal of Operations and Production Management, 1988.

-Li, Anlong.Co, Henry C. A dynamic programming model for the Kanban assignment problem in a multistage multiperiod production system. International Journal of Production Research, 1991.

-Raymond, Louis. Custom Kanban. Designing the system to meet the needs of your enviroment. Productivity Press, New York, 2006.

-Lummus, Rhonda R. A simulation analysis of sequencing alternatives for JIT lines using kanbans. Journal of Operations Management, 1995.

-Matta et.al. Analysis of assembly systems controlled with kanbans. European Journal of Operation Research, 2005.

-Mitra, Debasis.Mitrani, Isi. Analysis of a Kanban Discipline for Cell Coordination in Production Lines, I. Management Science, 1990.

-Mitra, Debasis.Mitrani, Isi. Analysis of a Kanban Discipline for Cell Coordination in Production Lines, II, Stochastic Demands. Operations Research, 1991.

-Mittal, S.Wang, Hsu-Pin (Ben). Simulation of JIT production to determine number of kanbans. International Journal of Advanced Manufacturing Technology, 1992.

-Mitwasi, M. G.Askin, R. G. Production planning for a multi-item, multi-stage Kanban system. International Journal of Production Research, 1994.

-Miyazaki, S.Ohta, H.Nishiyama, N. The optimal operation planning of Kanban to minimize the total operation cost. International Journal of Production Research, 1988. -Moeeni, F.Sanchez, S. M.Vakharia, A. J. A robust design methodology for Kanban system design. International Jo- urnal of Production Research, 1997.

-Ohno, K.Nakashima, K.Kojima, M. Optimal numbers of two kinds of kanbans in a JIT production system. International Journal of Production Research, 1995.

- Onvural, R. Survey of closed queueing networks with blocking. ACM Computing Surveys, 1990.

-Philipoom, Patrick R.Rees, Loren P.et.al. An investigation of the factors influencing the number of Kanbans required in the implementation of the JIT technique with Kanbans. International Journal of Production Research, .1987.

-Philipoom, Patrick R.Rees, Loren P.et.al. A mathematical programming approach for determining workcentre lotsizes in a just-in-time system with signal kanbans. International Journal of Production Research, 1990.

-Philipoom, Patrick R.Rees, Loren P.Taylor III, Bernard W. Simultaneously determining the number of kanbans, container sizes, and the final-assembly sequence of products in a just-in-time shop. International Journal of Production Research, 1996.

-Price, Wilson L.Gravel, Marc.et.al. Modelling the performance of a Kanban assembly shop. International Journal of Production Research, 1995.

-Rees, Loren P.Philipoom, Patrick R.et.al. Dynamically adjusting the number of kanbans in just-in-time production system using estimated values of leadtime. IIE Transactions, 1987.

-Rees, Loren P.Huang, P.Y.Taylor III, Bernard W. A comparative analysis of an MRP lot-for-system and a Kanban system or a multistage production operation. International Journal of Production Research, 1989.

-Sarker, Bhaba R.Harris, Roy D. The effect of imbalance in a just-in-time production system: A simulation study. International Journal of Production Research, 1988.

-Savsar, M. Effects of Kanban withdrawal policies and other factors on the performance of JIT systems - a simulation study. International Journal of Production Research, 1996.

-Schonberger, Richard J. Applications of Single-Card and Dual-Card Kanban. Interfaces, 1983.

-Singh, N.Brar, J. K. Modelling and Analysis of Just-inTime Manufacturing Systems: A Review. International Journal of Operations \& Production Management, 1992.

-Sipper, Daniel.Shapira, Reuven. JIT vs. WIP--a trade-off analysis. International Journal of Production Research, 1989. 
-Spearman, Mark L.Woodruff, David L.Hopp, Wallace J. CONWIP: a pull alternative to kanban. International Journal of Production Research, 1990.

-Sugimori, Y. Kusunoki, K. Uchikawa, S. Kusunoki, K.Uchikawa, S. Toyota production system and Kanban system Materialization of just-in-time and respect-for-human system. International Journal of Production Research, 1977.

-Suri, Rajan. How to plan and implement POLCA: A matrial control system for high-variety or custom-engineered products. Decision Sciences and Eng. Systems, 2003. -Sury, RoyStubitz, Steven J. Kanban and Just-In-Time at Toyota (BR). International Journal of Production Research, 1987.

-Takahashi, K.Muramatsu, Rintaro.et.al. Feedback method of production ordering system in multi-stage production and inventory systems. International Journal of Production Research, 1987.

-Takahashi, K. Determining the number of kanbans for unbalanced serial production systems. Computers and Industrial Engineering, 1994.

-Tayur, S. Structural properties and a heuristic for Kanban-controlled serial lines. Management Science, 1993.

-Uzsoy, Reha. Martin-Vega, Louis A. Kanban-based demand-pull systems: a survey and critique. Manufacturing Review, 1990.

-Vatalaro, James. Implementing a mixed model kanban system. The Lean replenishment technique for Pull production. Productivity press, NY. 2003.

-Wang, Hunglin.Wang, Hsu-Pin (Ben). Determining the number of kanbans: A step toward nonstock-production. International Journal of Production Research, 1990. 